\title{
Visual search enhances subsequent mnemonic search
}

\author{
Holly A. Westfall • Kenneth J. Malmberg
}

Published online: 8 September 2012

(C) Psychonomic Society, Inc. 2012

\begin{abstract}
We examined how the performance of a visual search task while studying a list of to-be-remembered words affects subsequent memory for those words by humans. Previous research had suggested that episodic context encoding is facilitated when the study phase of a memory experiment requires, or otherwise encourages, a visual search for the tobe-remembered stimuli, and theta-band oscillations are more robust when animals are searching their environment. Moreover, hippocampal theta oscillations are positively correlated with learning in animals. We assumed that a visual search task performed during the encoding of words for a subsequent memory test would induce an exploratory state that would mimic the one that is induced in animals when performing exploratory activities in their environment, and that the encoding of episodic traces would be improved as a result. The results of several experiments indicated that the performance of the search task improved free recall, but the results did not extend to yes-no or forced choice recognition memory testing. We propose that visual search tasks enhance the encoding of episodic context information but do not enhance the encoding of to-be-remembered words.
\end{abstract}

Keywords Memory $\cdot$ Recall $\cdot$ Recognition $\cdot$ Visual search

Performance on episodic-memory tasks, which require one to remember past experiences, is enhanced when memory is tested in the same context in which the memories were encoded (for a review, see Smith \& Vela, 2001). Such empirical findings implicate the theoretical relationship

H. A. Westfall $(\bowtie) \cdot$ K. J. Malmberg

Department of Psychology, University of South Florida,

4202 East Fowler Avenue, PCD 4118G,

Tampa, FL 33620, USA

e-mail: hwestfall@mail.usf.edu

K. J. Malmberg

e-mail: malmberg@usf.edu between the information that one seeks to encode or retrieve and the context in which such activities occur. While context-dependent memory has been the subject of much research, the mechanisms by which it occurs are still not well understood, at either the cognitive or the physiological level. Moreover, there is little consensus as to a model that could bridge these levels of our understanding of behavior. Drawing upon the literature on human and animal brain physiology, we noted a well-documented relationship between the activities of the individual and oscillatory activity in the brain in regions supporting episodic memory and the binding of items to the contexts in which they occur (Buzsáki, 2002). On the basis of the literature, we hypothesized that inducing human participants into a cognitive state of exploration when studying items would enhance episodic memory. Indeed, this is what we found, and we suggest that such activities enhance episodic memory as the result of enhanced binding of items to the contexts in which they occur.

In this article, we first review several investigations of theta-band oscillations in the brain. Next, we report the results of five experiments indicating that one factor known to induce theta oscillations in humans and animals corresponds to increased performance on free recall tasks, but not on recognition memory tasks. Finally, we describe several phenomena indicative of context-dependent memory at the behavioral level and speculate that the enhanced memory in our experiments was due to a more robust binding of items and context in their episodic-memory traces.

\section{Theta oscillations}

We tested the hypothesis that memory encoding is enhanced when humans explore their surroundings. Indeed, the correlation of exploratory activity and brain activity is extensively documented in animals and humans, and research has 
focused on brain oscillations in the theta frequency band, which are usually defined as oscillations occurring over an extended period of time in the 2- to $12-\mathrm{Hz}$ range (Buzsáki, 2002). While the precise function of theta oscillations is not yet well understood, hippocampal theta is closely related to learning and memory (e.g., Hasselmo, 2005). Theta is concentrated in two areas of the human brain: the cortex and the hippocampus. In animals, theta is associated with the hippocampus (Buzsáki, 2002). Berry and Thompson (1978) found that the rate of acquisition of classical eye blink conditioning is predicted by hippocampal theta activity measured 2 min before training; hippocampal oscillations in the theta range of $2-8 \mathrm{~Hz}$ predicted faster rates of learning than did oscillations in the nontheta range. This suggests that being in a state corresponding to high theta activity may enhance the encoding of memories.

Stronger evidence linking theta to learning has come from investigations of the correlation of theta oscillations during learning with conditioning. Seager, Johnson, Chabot, Asaka, and Berry (2002) reported that rabbits trained only during the presence of theta activity learned conditioning to criterion in half as many trials as those trained only during non-theta-frequency activity. Studies in which the natural occurrence of theta was impeded by lesions (Berry \& Thompson, 1979) or by drug infusion in the medial septum (Asaka, Seager, Griffin, \& Berry, 2000) found that the experimental group required significantly more trials to reach the conditioning criterion than did a control group. Conversely, Berry and Swain (1989) increased theta activity in rabbits by water deprivation. The experimental group learned eye blink conditioning to criterion in significantly fewer trials than did the control group and also had a greater percentage of electrical brain activity in the theta frequency. Thus, theta is correlated with enhanced learning, but it may not be necessary for learning to occur.

These findings suggest that activities associated with relatively high levels of theta power may also be associated with enhanced learning and memory. For instance, theta is also correlated with exploratory activities in rodents: Hippocampal theta is observed when they search their environment (Vanderwolf, 1969). However, theta is not observed when rodents are performing automatic, repetitive movements such as chewing, drinking, or grooming (Vanderwolf, 1969), indicating that bodily movements are not sufficient for increasing theta power. In fact, theta is also observed when passive, nonambulatory movements through a physical space are initiated by mechanical means (Foster, Castro, \& McNaughton, 1989). In humans, virtual navigation of rendered scenes induces coordinated hippocampal and cortical theta activity (Ekstrom et al., 2005), suggesting that physical relocation is not necessary to induce theta. Moreover, the richness of the environmental context does not appear to be important, as visual information is not necessary to observe the increase in theta associated with volitional movements in rats (Chen, Yang, Lin, \& Kuo, 2011). These findings, as a package, suggest that simply being in a mental state of exploration is sufficient for inducing theta activity.

\section{Eye movements}

While physical relocation is unnecessary to induce theta, it seems prudent to investigate whether simply moving the eyes may be sufficient to induce theta, as both virtual and physical navigation require eye movements in participants. Prior research has indicated that simply moving the eyes bilaterally between study and test can improve memory performance on a variety of tasks. Christman and Propper (2001) found that $30 \mathrm{~s}$ of saccadic horizontal eye movements aided free recall. Similar enhancements in memory have also been reported for associative recognition and item recognition (Christman, Garvey, Propper, \& Phaneuf, 2003; Parker, Relph, \& Dagnall, 2008), where accuracy was increased via both an increase in hit rates (HRs) and a decrease in false alarm rates (FARs), known as a mirror effect. Such eye movements should be distinguished from visual search, insofar as the goal of the participant in the first case is to move his or her eyes, whereas the goal in the second case is to locate a target. Thus, bilateral eye movements and visual search may affect memory in different manners. In addition, it is important to note that improvements in memory are observed only when the eye movements occur between learning and retrieval (Brunyé, Mahoney, Augustyn, $\&$ Taylor, 2009). The effect of eye movements during encoding has not been reported, although it is probably impossible to conduct a visual search without inducing eye movements as well.

The relationship between theta and bilateral eye movements is not yet clear. In a study using electroencephalography to investigate the physiological effects of $30 \mathrm{~s}$ of bilateral saccadic eye movements, Propper and colleagues found a decrease in gamma-frequency coherence across the two hemispheres of the brain (Propper, Pierce, Geisler, Christman, \& Bellorado, 2007). Gamma activity, like theta activity, is associated with episodic memory processes. However, Propper et al. found no significant change in theta coherence following the eye movement task. These findings present further evidence that bilateral eye movements and theta oscillations have independent effects on memory.

\section{Experiments 1 and 2}

On the basis of the aforementioned literature, we hypothesized that exploratory activity would be associated with 
enhanced human episodic memory, perhaps as the result of the creation of strong episodic associations between the tobe-remembered stimuli and the context in which learning occurred. To test this hypothesis, we developed a procedure that required participants in one condition to search for a word that was displayed in one of several computer screen locations. Following the search, the participants' task was to type in the word using the computer keyboard. Hence, the exploration task was similar to those in several previous experiments in the animal literature, insofar as the participant was not required to engage in a physical displacement or to execute a complex locomotive routine other than simple eye movements. However, the visual search was nontrivial, since the target locations were randomly determined and the color of the font in which the target appeared was only slightly darker than the white background of the display. In the control condition, a visual search of the display was unnecessary, because the words appeared in the same central location on each study trial; the participants' task here was simply to type the target word into the computer. This same basic procedure was used during the study phase of all experiments reported in this article.

For the first two experiments, memory was tested via free recall. In Experiment 1, relatively uncommon English nouns were used as the stimuli, on the assumption that rare words would provide a more challenging target for the visual search. For instance, rare words are more difficult to identify and name than are more common words (see Malmberg \& Nelson, 2003, for a review). In Experiment 2, we added a condition in which relatively common words were also tested, in order to replicate the results from Experiment 1 and to assess whether word frequency would interact with the visual search condition.

\section{Method}

Participants A group of 51 undergraduates at the University of South Florida participated in Experiment 1, and an additional 70 undergraduates at the University of South Florida took part in Experiment 2, both groups in exchange for course credit.

Materials and design In Experiment 1, all of the stimuli were nouns taken from Francis and Kučera (1982), with normative frequencies of 20-50/million. For each participant in Experiment 1, 72 words were randomly sampled from the pool of 540 words to create three 24-item lists. The participants were randomly assigned to one of two conditions. In both conditions, the words were displayed one at a time in light gray [RGB $(221,221,221)$, HSL $(160,0,208)]$ 18-point Tahoma font on the white background of a 15-in. flat-panel LCD monitor without a central fixation point. The participant was required to locate each word and to type it into a response box located in the center of the screen in order to move on to the next word. In the no-search condition, the words were always displayed in the center of the computer screen just above the response box. In the search condition, the words were randomly displayed in one of eight locations around the perimeter of the screen, including in each corner and between each of two adjacent corners. For each word list, following the study phase, the participants performed a 30-s distractor task in which they mentally added single digits presented one at a time on the computer screen. Then they were given $60 \mathrm{~s}$ to recall as many words as possible, in any order, by typing the words into a response box on the screen.

Experiment 2 was similar to Experiment 1, except that an additional condition was run in which words with normative frequencies greater than 50 per million were used. Experiment 2 used a $2 \times 2$ (Search $\times$ Word Frequency) mixed factorial design, with search condition remaining a betweensubjects variable and word frequency as a within-subjects variable. Each participant was required to learn four lists of 24 words (two of each frequency), with words blocked by word frequency and the presentation order counterbalanced between subjects.

Procedure The participants completed the experiment in individual booths on desktop PCs. All portions of the experiment, including the instructions and informed consent, were performed on the computers. Participants were informed before the start of the experiment that their memory would be tested for the words that they were about to see.

\section{Results and discussion}

The results are presented in Table 1. For Experiment 1, an independent samples $t$ test revealed a significantly greater proportion of items recalled in the search condition than in the no-search condition, $t(49)=2.36, p<.05$. For Experiment 2, a two-way ANOVA revealed a main effect of word frequency, $F(1,68)=14.28, p<.001, \eta_{\mathrm{p}}{ }^{2}=.174$, and a main effect of search, $F(1,68)=4.94, p<.05, \eta_{\mathrm{p}}{ }^{2}=.068$. Participants in the search condition outperformed those in the no-search condition, and higher-frequency words were

Table 1 Means and standard errors for Experiments 1 and 2

\begin{tabular}{|c|c|c|c|c|c|}
\hline & \multirow[t]{2}{*}{ WF } & \multicolumn{2}{|c|}{ No Search } & \multicolumn{2}{|c|}{ Search } \\
\hline & & $M$ & $S E$ & $M$ & $S E$ \\
\hline Experiment 1 & MF & .229 & .015 & .279 & .015 \\
\hline \multirow[t]{2}{*}{ Experiment 2} & MF & .222 & .016 & .278 & .015 \\
\hline & $\mathrm{HF}$ & .268 & .021 & .317 & .021 \\
\hline
\end{tabular}

$W F$ word frequency; $M F$ medium frequency; $H F$ high frequency 
more likely to be recalled than lower-frequency words. The Frequency $\times$ Search interaction was not reliable, $F<1$. Inspection of Table 1 indicates that the means and standard errors for medium-frequency nouns in both experiments were nearly identical. Thus, Experiment 2 replicated and extended the results of Experiment 1 to higher-frequency words.

The amount of time spent searching memory might be informative as to the effects of visual search on metacognitive control and motivation during retrieval. Therefore, we also collected data concerning the rates of output for Experiment 2. If the search task caused an increase in context binding, we might also expect to see an increase in the familiarity of the items and, thus, an increase in the amount of time spent free-recalling (Malmberg, 2008). Accordingly, we examined the time of first-word recall, the time of lastword recall, and the rate of output across search and word frequency. For rate of output, there was no effect of word frequency, $F(1,68)=2.608, p=.111, \eta_{\mathrm{p}}{ }^{2}=.037$, search was marginally significant, $F(1,68)=2.850, p=.096, \eta_{\mathrm{p}}{ }^{2}=$ .040 , and the Word Frequency $\times$ Search interaction was not significant, $F<1$. High-frequency words were output at a slightly faster rate in the search condition than in the other conditions. There was no effect of word frequency on the speed of the first-item output, $F<1$, or on search, $F<1$, and the Word Frequency $\times$ Search interaction was marginally significant, $F(1,68)=3.242, p=.076, \eta_{\mathrm{p}}{ }^{2}=.046$. Therefore, we performed a paired-samples $t$ test to compare medium- and high-frequency words in the search condition and found a significant difference, $t(35)=2.217, p=.041$; the first highfrequency word was output more quickly than the first medium-frequency word in the search condition. Finally, we looked at the overall amount of time spent outputting words. There was a main effect of search, $F(1,68)=4.077, p=.047$, $\eta_{\mathrm{p}}{ }^{2}=.057$, word frequency was found to be marginally significant, $F(1,68)=2.907, p=.093, \eta_{\mathrm{p}}{ }^{2}=.041$, and the Word Frequency $\times$ Search interaction was not significant, $F(1$, $68)=1.053, p=.308, \eta_{\mathrm{p}}{ }^{2}=.015$. We performed a pairedsamples $t$ test to compare medium- and high-frequency words in the search condition and found a significant difference in the overall amount of time outputting words, $t(35)=$ 2.072, $p=.046$. To summarize these findings, we found a slight advantage for high-frequency words in the rate of recall and the amount of time spent retrieving them in the search condition. This is consistent with the fact that the number of words recalled was greatest in this condition, but it does not appear that the search task itself had a direct influence on the rate or the amount of time spent retrieving.

\section{Experiment 3}

With no detectable difference in the effect of free recall of high- versus medium-frequency words, we more directly investigated how the difficulty of the visual search task was related to episodic memory performance. In this experiment, we varied the difficulty of the search by using a visual precuing procedure in one condition, in which the location of the to-be-remembered word was precued with a red rectangle on the display. By incorporating a precue, we attempted to guide eye movement toward the stimulus, thereby facilitating search for the study word by reducing the space in which a participant must direct attention. In visual search tasks, precuing the location of a stimulus results in greater accuracy in the identification of the stimulus and/or a shorter reaction time (e.g., Zhuang \& Papathomas, 2011). Thus, if the difficulty of the search determines how well items are encoded, there should be a main effect of the precue. On the other hand, being in a state of exploration may itself be sufficient to enhance the encoding of new memories, just as being in a state of high theta power enhances conditioning (cf. Berry \& Thompson, 1978). If so, we would expect to observe a significant effect of the search, but not a significant effect of the precue.

\section{Method}

Participants A group of 121 undergraduates at the University of South Florida took part in the experiment in exchange for course credit.

Materials, design, and procedure The materials, design, and procedure were identical to those of Experiment 1, with the following exceptions: We noticed upon further investigation that participants in the search condition took an average of about $300 \mathrm{~ms}$ longer than those in the no-search condition to locate each word and type it into the computer. In an attempt to better equate this retention interval, we used four lists of 12 medium-frequency nouns. The search variable became a within-subjects variable, and word lists were blocked by search condition. To better control eye movements, we also incorporated a between-subjects precue condition. In the precue condition, a small red square, about the height and width of a lowercase letter, appeared for $500 \mathrm{~ms}$ before the presentation of the word. The precue remained in place just to the left of each word during study. This precue would require participants to move their eyes toward the word and would facilitate the search process. In addition, to make the task slightly more difficult, we made the font color for the study words in all conditions one shade lighter [RGB (238, $238,238), \operatorname{HSL}(160,0,224)]$. In the study instructions, we also emphasized that participants should respond as quickly as possible. If a participant did not respond within $15 \mathrm{~s}$ after a study word was displayed, a warning screen appeared urging them to respond more quickly. If the warning screen displayed, the reaction time for that trial was recorded as 999, and the next trial began. 
Results and discussion

The results are presented in Table 2. A $2 \times 2$ (Precue $\times$ Search) mixed factorial ANOVA revealed a main effect of search, $F(1,119)=5.37, p<.05, \eta_{\mathrm{p}}{ }^{2}=.043$, consistent with our previous free recall results. The effect of the precue was not significant, $F<1$, nor was the Search $\times$ Precue interaction, $F<1$. To determine whether the addition of the precue aided the search process, we compared the reaction time data across the precue conditions for the study lists in the search condition and found a significant effect of precue, $t$ $(119)=4.297, p<.001$. Reaction times for study words in the precue condition $(M=3.53, S D=1.18)$ were faster than those for study words in the no-precue condition $(M=4.45$, $S D=1.18)$. This decrease in reaction times for precued words is consistent with the literature on visual search and demonstrates that the addition of the precue did indeed facilitate the search process for participants. These results suggest that being in a state of active search enhances memory, regardless of the difficulty of the task.

\section{Experiments 4 and 5}

In the final two experiments, we investigated two mechanisms by which visual search may enhance episodic memory. One way for visual search during study to enhance episodic memory is by enhancing the encoding of the target items. For recall, enhanced item encoding would improve recovery of partial information stored during study and/or allow items recalled earlier during the free recall sequence to serve as better retrieval cues (e.g., Malmberg \& Shiffrin, 2005; Raaijmakers \& Shiffrin, 1981). Enhanced encoding of item information should also improve recognition memory. According to some models, this would produce increases in HRs and decreases in FARs (see, e.g., McClelland \& Chappell, 1998; Shiffrin \& Steyvers, 1997), whereas other models predict an increase in hit rates only (e.g., Gillund \& Shiffrin, 1984).

Another possibility is that visual search may more strongly bind items to the study context. Traditionally, context effects are more robust in free recall than in recognition memory (Smith, 1988). Since the participant has only the context cue with which to initially probe memory in free

Table 2 Means and standard errors for Experiment 3

\begin{tabular}{llllll}
\hline & \multicolumn{2}{l}{ No Search } & & & \multicolumn{2}{l}{ Search } & \\
\cline { 2 - 3 } \cline { 5 - 6 } & $M$ & & & $M$ & \multicolumn{2}{l}{$S E$} \\
\hline No precue & .463 & .021 & & .491 & .019 \\
Precue & .442 & .019 & & .468 & .018 \\
\hline
\end{tabular}

recall, context plays a central role in the memory access. For recognition, the test item is also used to probe memory. Moreover, the context used to probe memory is paired with both targets and foils. According to global-matching models, both targets and foils should match the contexts of memory better when context is stored relatively well (cf. Murnane, Phelps, \& Malmberg, 1999). If so, the familiarity of both targets and foils should be enhanced when a visual search is conducted during study, and the predicted result would be that both HRs and FARs should be greater in the search than in the no-search condition for Experiment 4, which used a yes-no recognition procedure. Experiment 5 used a two-alternative forced choice procedure, and the corresponding prediction was that no difference in recognition accuracy should be observed.

Method

Participants One group of 38 undergraduates at the University of South Florida participated in Experiment 4, and another of 34 students at the University of South Florida took part in Experiment 5, both in exchange for course credit.

Materials, design, and procedure The materials, design, and procedure were identical to those used in Experiment 1 with the following exceptions: Three study lists each contained 75 medium-frequency nouns. Following each study list and distractor task, participants completed an old/new recognition task in Experiment 4 and a two-alternative forced choice task in Experiment 5. At test, all 75 previously studied targets, as well as 75 unstudied foils, were presented in a random order. The test words were always displayed in the center of the screen, regardless of the placement of the word at study. Participants indicated their responses with the " 0 " and " 1 " keys on the number pad.

Results and discussion

The HR, FAR, and HR - FAR results from Experiment 4 are presented in Table 3. Both the HRs and the FARs were significantly greater in the search condition $[\mathrm{HR}, t(36)=2.95, p<$ .05 ; FAR, $t(36)=2.15, p<.05]$. There was no significant effect of the search on the difference between the HRs and FARs. Thus, recognition accuracy was unaffected by the search. This

Table 3 Hit rates (HR) and false alarms (FAR) for Experiment 4

\begin{tabular}{|c|c|c|c|c|c|c|}
\hline & \multicolumn{2}{|l|}{ HR } & \multicolumn{2}{|l|}{ FAR } & \multicolumn{2}{|c|}{ HR - FAR } \\
\hline & $M$ & $S E$ & $M$ & $S E$ & $M$ & $S E$ \\
\hline No search & .573 & .038 & .149 & .031 & .424 & .043 \\
\hline Search & .707 & .026 & .246 & .033 & .461 & .034 \\
\hline
\end{tabular}


conclusion is supported by the results of Experiment 5, which used a forced choice procedure. There was no significant difference in the proportions correct in recognition trials between the search condition $(M=.80, S D=.09)$ and the nosearch condition $(M=.80, S D=.09), t(32)=0.02, p=.986$. The results of both experiments were that visual search had little or no impact on recognition accuracy. This is not simply a null result to be dismissed by the skeptic on the basis of frequentist theory, since a significant effect of the search task on HRs and FARs did occur in Experiment 4. However, since HRs and FARs were similarly affected by the search task, no change in overall accuracy was obtained.

\section{General discussion}

When one tests memory via free recall, participants who are required to search for the study material outperform participants who do not need to search for the study material. The difficulty of the search task has little impact; similar enhancements in free recall were observed when the location of the target was precued. The visual search task does not improve recognition memory, but it is associated with increases in both HRs and FARs that are about equal and quite substantial. The interaction between search and task is different from that reported in the eye-movement literature, in which both recall and recognition have been found to be enhanced when a study list is followed by an interval of bilateral eye movements prior to testing (Brunyé et al., 2009; Christman et al., 2003, Christman \& Propper, 2001; Parker et al., 2008). The recognition results indicate that visual search does not increase the strength with which items are encoded. It is, however, possible that the visual search task enhanced the encoding of the episodic context by improving the ability of the participant to accurately reinstate the study context when initiating free recall, whereas increasing contextual strength should enhance the familiarity of both targets and foils about equally during recognition testing, producing no benefits for two-alternative forced choice testing or for $d^{\prime}$ in yes-no testing.

\section{Context effects in free recall}

Though much research has implicated the role of context information in episodic memory, what context is and how it is used to perform episodic-memory tasks is not very well understood. Here we may define context information as that information concerning the state of the participant that is correlated between successive events. For instance, temporal context may include information about physiological states, mood states, and a person's external environment that persist during the course of a series of learning (or test) trials. Hence, the correlations between events stored in an experimental setting are often temporal correlations, as well. The key to this definition of temporal context is that it changes very little, if at all, over a series of trials. Therefore, when a series of items are studied, these contextual representations are associated with each trace stored to represent the occurrence of an item, unless for some reason a large change in temporal context is caused by the experimenter, another external source, or the participant's own volition (e.g., Lehman \& Malmberg, 2009, 2011; Sahakyan \& Kelley, 2002). ${ }^{1}$

Changes in context have a negative effect on episodic memory. For example, context-dependent memory effects are commonly found when memory is tested via free recall with a variety of temporal-context manipulations, including the state of sobriety (Goodwin, Powell, Bremer, Hoine, \& Stern, 1969), mood (Eich \& Metcalfe, 1989), and the environment (Godden \& Baddeley, 1975).

When participants are asked to generate the items from study, all that is initially available to probe memory is the test context. Hence, the match of the context used to probe memory and the context stored during test largely determines the effectiveness of these initial probes during free recall (cf. Lehman \& Malmberg, 2009). Note that in most models of memory there are two potential sources of context-dependent memory for the free recall task. The first source is the context reinstatement attempted by the participant in order to initiate free recall, and this may be more difficult when the contexts have changed between study and test. The second source of context-dependent free recall, related to the first, is the match between the contents of the reinstated context cue and the context stored in memory. This match may be lower when the context has changed between study and test. If so, interference from irrelevant, nontarget memory traces would be greater following a change in context. In terms of the present findings, we have proposed that visual search enhances the encoding of context during study, thereby making it easier to reinstate at test, since there is little reason to suspect that the visual search task causes a greater change in context between study and test than does the control condition.

\section{Context effects in recognition}

Traditionally, context-dependent memory is more easily found in free recall tasks than in recognition tasks (Smith, 1988), and this has been a challenge to understand. One difference between free recall studies and recognition memory studies is that in the recognition memory studies, context is almost always operationally defined as the environmental context, and the manipulations of environmental context vary in the

\footnotetext{
${ }^{1}$ Item and context information may also be distinguished by the time courses of their encoding in long-term traces (Malmberg \& Shiffrin, 2005).
} 
amount of the environment that is changed. Thus, the effect of context change on recognition is somewhat vexing, due to the variability in the extents to which and the manners in which context change affects recognition accuracy. For example, Murnane, Phelps, and Malmberg (1999) consistently found very small context effects, while more recently, Hockley (2008) found substantial context effects for recognition.

Whereas at first glance, the recognition procedures used in such experiments seem very similar, there are several differences. In recognition experiments in which relatively large context effects have been reported, in the form of same-direction changes in both HRs and FARs, context has been operationally defined as the photograph in which a word is embedded (e.g., Hockley, 2008). In other experiments, a more impoverished manipulation of the color of the background while displaying a word on the computer screen has been used, and the increases in HRs and FARs have been much smaller (Murnane \& Phelps, 1993). While one might suppose that the differences in the magnitudes of these context manipulation are what has produced differences in the magnitudes of the context effects, more elaborate and interesting context scenes may have encouraged their exploration, producing larger context effects, whereas studies in which a relatively impoverished background context was used may not have encouraged an exploration, and therefore produced relatively small context effects (e.g., Murnane \& Phelps, 1995).

In any case, changes in context often produce increases in HRs and FARs much like those observed here (cf. Hockley, 2008; Murnane \& Phelps, 1993), and such changes are consistent with the hypothesis that visual search enhances contextual encoding. ${ }^{2}$ According to models like the "item, context, and ensemble" model (ICE; Murnane et al., 1999), increases in HRs and FARs are the results of a better match between the associated context of the retrieval cue and the context stored in memory. The enhanced-encoding-of-context hypothesis is similar, in that the performance of the visual search task enhances the encoding of context information in long-term episodic traces. When the study context is reinstated and used to probe memory along with the stimulus during recognition testing, the greater match between the context in the retrieval cue and the context stored in memory enhances the familiarity of the test items, regardless of whether the stimulus was a target or a foil.

ICE assumes that encoding results in an integration or association of item and context information that is independent of the individual representations of the item and the context-referred to in ICE as an ensemble-under some

\footnotetext{
$\overline{2}$ Same-direction changes in HRs and FARs are also consistent with a shift in response bias. There is no theoretical reason why bias should be determined by search activity during study, but nevertheless, different mechanisms may produce the changes in free recall and recognition performance.
}

conditions. Thus, an ensemble formed from an item and the context in which it occurs plays a role similar to that played by a convolution or association between two items in TODAM (Murdock, 1982). When participants perform a task that requires the integration of a to-be-remembered item and the context in which it occurred, an ensemble of information is encoded that will only match a retrieval cue composed of the same item and context information. Because an ensemble formed from an item and context is independent of the item and the context itself, an ensemble produces relatively good matches between a retrieval cue and memory for targets but not for foils. Accordingly, the storage and probe with an ensemble of item and context information produces increases in HRs, but no increase in FARs (i.e., an increase in recognition accuracy). ${ }^{3}$ Such context-dependent recognition memory has been observed when participants are instructed to actively relate or associate the items and the context in which they occur during study (Murnane et al., 1999). Murnane et al. reported that recognition accuracy was greater in the same-context condition than in the different-context condition only when the context was operationally defined as a computer-rendered scene and participants were instructed to integrate the to-beremembered word with the scene in which it appeared. Importantly, such results are inconsistent with the increases in HRs and FARs that we observed for yes-no recognition. However, the rendered scenes used by Murnane et al. were meaningful stimuli, as compared to the white background screens that we used here. In the case of the present experiments, we were therefore unlikely, according to ICE, to see an increase in context-dependent discrimination, as we would expect approximately equal shifts in bias for HRs and FARs, since we did not use contextually rich stimuli in our manipulations or instruct participants to create ensembles.

\section{Conclusions}

We found that the performance of a visual search task during encoding enhances free recall but has no impact on the accuracy of recognition memory. The present experiments were motivated by several findings from the animal learning literature and the cognitive neuroscience literature. Although we did not record brain activity in the present experiments, one might presume that the search activity was correlated with theta-band oscillations in brain activity, on the basis of the finding from prior animal studies that enhanced encoding would

\footnotetext{
${ }^{3}$ One way to formally describe the relationship between item, context, and the ensemble is to use item vector $\mathbf{A}$ and context vector $\mathbf{B}$, and to represent the ensemble by the convolution of $\mathbf{A}$ and $\mathbf{B}$, or $\mathbf{A} * \mathbf{B}$ (cf. Murdock, 1982).
} 
be the result of the induction of theta activity that is typically observed during exploratory activities. Our findings suggest that visual exploration in humans enhances the encoding of context in episodic-memory traces. Perhaps theta is associated with enhanced contextual encoding, as well. Tenuous as the relationship between visual search activity and human brain functioning may be, our findings encourage us to speculate about the role, if any, that theta may have in learning (e.g., Burgess \& O'Keefe, 2005; Hasselmo, 2005). Although we cannot add much with confidence to the discussion about the role of theta in learning, we have used this literature as a means to discover a novel way to enhance some forms of episodic memory, a goal that up to this point has been largely elusive in the literature on human memory.

\section{References}

Asaka, Y., Seager, M. A., Griffin, A. L., \& Berry, S. D. (2000). Medial septal microinfusion of Scopolamine disrupts hippocampal activity and trace jaw movement conditioning. Behavioral Neuroscience, 114, 1068-1077.

Berry, S. D., \& Swain, R. A. (1989). Water deprivation optimizes hippocampal activity and facilitates nictitating membrane conditioning. Behavioral Neuroscience, 103, 71-76.

Berry, S. D., \& Thompson, R. F. (1978). Prediction of learning rate from the hippocampal electroencephalogram. Science, 200, 1298-1300.

Berry, S. D., \& Thompson, R. F. (1979). Medial septal lesions retard classical conditioning of the nictitating membrane response in rabbits. Science, 205, 209-211.

Brunyé, T. T., Mahoney, C. R., Augustyn, J. S., \& Taylor, H. A. (2009). Horizontal saccadic eye movements enhance the retrieval of landmark shape and location information. Brain and Cognition, 70, 279-288. doi:10.1016/j.bandc.2009.03.003

Burgess, N., \& O'Keefe, J. (2005). The theta rhythm. Hippocampus, 15, 825-826. doi:10.1002/hipo.20111

Buzsáki, G. (2002). Theta oscillations in the hippocampus. Neuron, 33, 325-340.

Chen, C. Y., Yang, C. C. H., Lin, Y. Y., \& Kuo, T. B. J. (2011). Locomotion-induced hippocampal theta is independent of visual information in rats during movement through a pipe. Behavioural Brain Research, 216, 699-704.

Christman, S. D., Garvey, K. J., Propper, R. E., \& Phaneuf, K. A. (2003). Bilateral eye movements enhance the retrieval of episodic memories. Neuropsychology, 17, 221-229.

Christman, S. D., \& Propper, R. E. (2001). Superior episodic memory is associated with interhemispheric processing. Neuropsychology, $15,607-616$

Eich, E., \& Metcalfe, J. (1989). Mood dependent memory for internal versus external events. Journal of Experimental Psychology: Learning, Memory, and Cognition, 15, 443-455.

Ekstrom, A. D., Caplan, J. B., Ho, E., Shattuck, K., Fried, I., \& Kahana, M. K. (2005). Human hippocampal theta activity during virtual navigation. Hippocampus, 15, 881-889.

Foster, T. C., Castro, C. A., \& McNaughton, B. L. (1989). Spatial Selectivity of rat hippocampal neurons: Dependence on preparedness for movement. Science, 244, 1580-1582.

Francis, W. N., \& Kučera, H. (1982). Frequency analysis of English usage: Lexicon and grammar. Boston, MA: Houghton Mifflin.
Gillund, G., \& Shiffrin, R. M. (1984). A retrieval model for both recognition and recall. Psychological Review, 91, 1-67. doi:10.1037/0033-295X.91.1.1

Godden, D. R., \& Baddeley, A. D. (1975). Context-dependent memory in two natural environments: On land and underwater. British Journal of Psychology, 66, 325-331. doi:10.1111/j.20448295.1975.tb01468.x

Goodwin, D. W., Powell, B., Bremer, D., Hoine, H., \& Stern, J. (1969). Alcohol and recall: State-dependent effects in man. Science, 163, $1358-1360$.

Hasselmo, M. E. (2005). What is the function of hippocampal theta rhythm? Linking behavioral data to phasic properties of field potential and unit recording data. Hippocampus, 15, 936-949.

Hockley, W. E. (2008). The effects of environmental context on recognition memory and claims of remembering. Journal of Experimental Psychology: Learning, Memory, and Cognition, 34, 1412-1429. doi:10.1037/a0013016

Lehman, M., \& Malmberg, K. J. (2009). A global theory of remembering and forgetting from multiple lists. Journal of Experimental Psychology: Learning, Memory, and Cognition, 35, 970-988. doi:10.1037/a0015728

Lehman, M., \& Malmberg, K. J. (2011). Overcoming the effects of intentional forgetting. Memory \& Cognition, 39, 335-347. doi:10.3758/s13421-010-0025-4

Malmberg, K. J. (2008). Recognition memory: A review of the critical findings and an integrated theory for relating them. Cognitive Psychology, 57, 335-384. doi:10.1016/j.cogpsych.2008.02.004

Malmberg, K. J., \& Nelson, T. O. (2003). The word frequency effect for recognition memory and the elevated-attention hypothesis. Memory \& Cognition, 31, 35-43. doi:10.3758/ BF03196080

Malmberg, K. J., \& Shiffrin, R. M. (2005). The "one-shot" hypothesis for context storage. Journal of Experimental Psychology: Learning, Memory, and Cognition, 31, 322-336.

McClelland, J. L., \& Chappell, M. (1998). Familiarity breeds differentiation: A subjective-likelihood approach to the effects of experience in recognition memory. Psychological Review, 105, 724-760. doi:10.1037/0033-295X.105.4.734760

Murdock, B. B., Jr. (1982). A theory for the storage and retrieval of item and associative information. Psychological Review, 89, 609626. doi:10.1037/0033-295X.89.6.609

Murnane, K., \& Phelps, M. P. (1993). A global activation approach to the effect of changes in environmental context on recognition. Journal of Experimental Psychology: Learning, Memory, and Cognition, 19, 882-894. doi:10.1037/0278-7393.19.4.882

Murnane, K., \& Phelps, M. P. (1995). Effects of changes in relative cue strength on context-dependent recognition. Journal of Experimental Psychology: Learning, Memory, and Cognition, 21, 158-172. doi:10.1037/0278-7393.21.1.158

Murnane, K., Phelps, M. P., \& Malmberg, K. (1999). Contextdependent recognition memory: The ICE theory. Journal of Experimental Psychology. General, 128, 403-415. doi:10.1037/ 0096-3445.128.4.403

Parker, A., Relph, S., \& Dagnall, N. (2008). Effects of bilateral eye movements on the retrieval of item, associative, and contextual information. Neuropsychology, 22, 136-145. doi:10.1037/ 0894-4105.22.1.136

Propper, R. E., Pierce, J., Geisler, M. W., Christman, S. D., \& Bellorado, N. (2007). Effect of bilateral eye movements on frontal interhemispheric gamma EEG coherence. The Journal of Nervous and Mental Disease, 195, 785-788.

Raaijmakers, J. G. W., \& Shiffrin, R. M. (1981). Search of associative memory. Psychological Review, 88, 93-134. doi:10.1037/ 0033-295X.88.2.93 
Sahakyan, L., \& Kelley, C. M. (2002). A contextual change account of the directed forgetting effect. Journal of Experimental Psychology: Learning, Memory, and Cognition, 28, 1064-1072.

Seager, M. A., Johnson, L. D., Chabot, E. S., Asaka, Y., \& Berry, S. D. (2002). Oscillatory brain states and learning: Impact of hippocampal theta-contingent training. Proceedings of the National Academy of Sciences, 99, 1616-1620. doi:10.1073/ pnas.032662099

Shiffrin, R. M., \& Steyvers, M. (1997). A model for recognition memory: REM - retrieving effectively from memory. Psychonomic Bulletin \& Review, 4, 145-166. doi:10.3758/ BF03209391
Smith, S. M. (1988). Environmental context-dependent memory. In G. M. Davies \& D. M. Thomson (Eds.), Memory in context: Context and memory (pp. 13-34). New York, NY: Wiley.

Smith, S. M., \& Vela, E. (2001). Environmental context-dependent memory: A review and meta-analysis. Psychonomic Bulletin \& Review, 8, 203-220. doi:10.3758/BF03196157

Vanderwolf, C. H. (1969). Hippocampal electrical activity and voluntary movement in the rat. Electroencephalography and Clinical Neurophysiology, 26, 407-418.

Zhuang, X., \& Papathomas, T. V. (2011). Cue relevance effects in conjunctive visual search: Cueing for location, color, and orientation. Journal of Vision, 11(7), 6:1-13. doi:10.1167/11.7.6 